\title{
A 3 Years Review of Hysteroscopy in a Private Hospital in Nigeria
}

\author{
${ }^{1,2}$ Okohue JE, ${ }^{1,2}$ Onuh SO, ${ }^{1}$ Akaba GO, ${ }^{1,2}$ Shaibu I, ${ }^{1,2}$ Wada I, ${ }^{3}$ Ikimalo J \\ ${ }^{1}$ Nordica Fertility Center @ Nisa Premier Hospital, Jabi Abuja, Nigeria \\ ${ }^{2}$ Port Harcourt Fertility Center, Port Harcourt, Rivers State, Nigeria \\ ${ }^{3}$ Department of Obstetrics and Gynecology, University of Port Harcourt Teaching Hospital, Rivers State, Nigeria
}

Correspondence: Jude Okohue, Port Harcourt Fertility Center @ Prime Medical Consultants. 4 Prime Close Rumuogba, Port Harcourt, Rivers State, Nigeria, E-mail: judosca@yahoo.com

\begin{abstract}
Background: Hysteroscopy is a valuable tool in the evaluation and treatment of infertility and many other gynecological procedures. Its use has relegated blind procedures for the investigation and treatment of endometrial pathologies and in some instances obviates the need for open surgeries. Unfortunately the equipment is only available in a few private hospitals in Nigeria.
\end{abstract}

Objective: To describe our experience with hysteroscopy in a private hospital setting in Nigeria.

Material and Methods: Retrospective reviews of all cases of hysteroscopy done between January 2003 and December 2005 at Nisa Premier Hospital (Nordica Fertility Centre), a dedicated fertility center in Abuja, Nigeria was carried out. Relevant information was extracted from the patients $\mathrm{case}$ notes and theater records. The hysteroscopic procedures were carried out during the proliferative phase of the patients menstrual cycle. All but nine patients had paracervical block with xylocaine in addition to intravenous pentazocine and promethazine for pain relieve. Normal saline was used as distention medium.

Results: A total of 87 hysteroscopies were carried out during the period under review. Eighty five patients $(97.7 \%)$ presented with infertility. Forty four patients (48.3\%) had single pathology while 23 (26.4\%) had multiple pathologies. The commonest pathology was intrauterine adhesions (64.2\%). There were 3 cases $(3.5 \%)$ of retained fetal bones within the endometrial cavity. Sixty four (73.6\%) patients underwent hysteroscopic surgical procedures. The commonest surgical procedure performed was intrauterine adhesiolysis $(67.2 \%)$, followed by polypectomy (35.9\%). One complication occurred during the study period (anesthetic).

Conclusion: Hysteroscopy is a valuable tool in the assessment of the uterine cavity. The equipments and skills are however presently lacking in Nigeria.

Keywords: Hysteroscopy, infertility, adhesiolysis.

\section{INTRODUCTION}

Hysteroscopy involves the passage of a small diameter telescope either flexible or rigid, through the cervix to directly inspect the uterine cavity.
It can be used for both diagnostic and operative purposes and remains a valuable tool in the evaluation and treatment of infertility, recurrent pregnancy loss and abnormal and postmenopausal uterine bleeding. It is also useful when saline infusion sonography findings are equivocal. ${ }^{1}$

Different distention media are available for both diagnostic and operative hysteroscopy. Among these are high viscosity dextran 70 and low viscosity fluids such as hypotonic, electrolyte free and isotonic, electrolyte containing solutions such as glycine $1.5 \%$ and normal saline respectively. A flexible hysteroscope which is used as an outpatient procedure commonly uses carbon dioxide as a distention medium. Although carbon dioxide is not used in the presence of uterine bleeding mainly because of the high-risk of embolism, many gynecologist still prefer it for its optimal clarity and patient comfort during insufflations for diagnostic hysteroscopy. ${ }^{2}$

Complications following hysteroscopy could be procedure related, media related or postoperative. ${ }^{1}$ The most common procedure related complication is uterine perforation. ${ }^{3}$ Cervical injuries could also occur. Media related complications include anaphylactic shock, noncardiogenic pulmonary edema; common with high viscosity fluids and gas embolism with carbon dioxide. Postoperative ascending infections, hematometria and pregnancy following endometrial ablation could also occur. ${ }^{1}$

\section{OBJECTIVES}

To describe our experience with hysteroscopy in a private hospital setting in Nigeria.

\section{MATERIAL AND METHODS}

Retrospective reviews of all cases of hysteroscopy done between January 2003 and December 2005 at Nisa Premier Hospital (Nordica Fertility Center), a dedicated fertility center in Abuja, Nigeria was carried out. Relevant information was extracted from the patients' case notes and theater records. 
A $4 \mathrm{~mm} 0^{\circ}$ rigid telescope was used. The hysteroscopic procedures were carried out during the proliferative phase of the patients' menstrual cycle. All but nine patients had paracervical block with xylocaine in addition to intravenous pentazocine and promethazine for pain relieve. The nine patients had general anesthesia as they had combined hysteroscopy and laparoscopy. Normal saline was used as distention medium. Therapeutic procedures were performed with hysteroscopic grasping forceps and scissors.

\section{RESULTS}

A total of 87 hysteroscopies were carried out during the period under review. Eighty five (97.7\%) patients presented with infertility. The parity ranged from $0-5$. Table 1 shows the age distribution of the patients. The age range was 24-47 years (35.5 \pm 4.7 ). Majority of the patients were aged between 35 and 39 years $(39.1 \%)$. As shown in Table 2, sixty three (72.4\%) patients were nulliparous. Among the nulliparous patients, 14 (22.2\%) had primary infertility. Seven of the fourteen patients (50\%) had intrauterine adhesions at hysteroscopy.

Twenty patients (23\%) had normal hysteroscopic findings. As shown in Table 3, forty four (48.3\%) patients had single pathology while 23 (26.4\%) had multiple pathologies. The commonest pathology was intrauterine adhesions (43 or 64.2\%). There were 3 (3.5\%) cases of retained fetal bones within the endometrial cavity. Table 4 shows the different hysteroscopic procedures carried out. Sixty four (73.6\%) patients underwent hysteroscopic surgical procedures. The commonest procedure performed was adhesiolysis (43 or $67.2 \%$ ) followed by polypectomy (23 or $35.9 \%)$. While $21(48.8 \%$ ) patients had an intrauterine contraceptive device (IUCD), 4 (9.3\%) had Pediatric Foley's catheter inserted following adhesiolysis. One complication (1.2\%) occurred during the study period and

Table 1: Age distribution of patients

\begin{tabular}{ccc} 
Age (years) & $n$ & $\%$ \\
$20-24$ & 1 & 1.1 \\
$25-29$ & 10 & 11.5 \\
$30-34$ & 24 & 27.6 \\
$35-39$ & 34 & 39.1 \\
$\geq 40$ & 18 & 20.7 \\
\hline Total & $\mathbf{8 7}$ & 100
\end{tabular}

Table 2: Parity distribution of patients

\begin{tabular}{ccc} 
Parity & $n$ & $\%$ \\
0 & 63 & 72.4 \\
1 & 15 & 17.2 \\
$2-4$ & 6 & 6.9 \\
$\geq 5$ & 3 & 3.5 \\
\hline Total & $\mathbf{8 7}$ & $\mathbf{1 0 0}$
\end{tabular}

Table 3: Findings at hysteroscopy

\begin{tabular}{lrr} 
& 3A: Single pathology & \\
Pathology & $n$ & $\%$ \\
IUA only & 25 & 28.7 \\
Endometrial polyp & 10 & 11.5 \\
Bone pieces & 3 & 3.5 \\
Cervical stenosis & 3 & 3.5 \\
Submucous fibroid & 2 & 2.3 \\
False passage & 1 & 1.1 \\
\hline Total & $\mathbf{4 4}$ & $\mathbf{5 0 . 6}$ \\
\hline \multicolumn{3}{c}{ 3B: Multiple pathologies } \\
Pathology & $n$ & \\
IUA and polyp & 8 & \\
IUA and cervical stenosis & 7 & 8.2 \\
Cervical stenosis and Endo. polyp & 5 & 5.7 \\
IUA and uterine perforation & 3 & 3.5 \\
\hline Total & $\mathbf{2 3}$ & $\mathbf{2 6 . 5}$ \\
\hline IUA = Intrauterine adhesions & \\
Endo. polyp = Endometrial polyp &
\end{tabular}

Table 4: Hysteroscopic procedures

$\begin{array}{lrr}\text { Procedure } & n & \% \\ \text { Adhesiolysis } & 43 & 67.2 \\ \text { Polypectomy } & 23 & 35.9 \\ \text { Cervical dilatation } & 15 & 23.4 \\ \text { Removal of bone piece } & 3 & 4.1 \\ \text { Submucous myomectomy } & 1 & 1.6\end{array}$

involved a 33 years old woman who developed apnea following completion of the procedure under general anesthesia.

\section{DISCUSSION}

During the last few years, diagnostic hysteroscopy has become a standard procedure in the diagnosis of fertility disorders, abnormal uterine bleeding and sonographically suspicious endometrial reflection. ${ }^{4}$ Today, hysteroscopic resection of uterine myomas, dissection of uterine septa and endometrial ablation are standard procedures. ${ }^{4}$ Performed concomitantly with laparoscopy, hysteroscopy becomes the most effective technique for evaluation of the uterine and tubal conditions that may play a role in female fertility. ${ }^{5}$

As would be expected being a fertility center, majority of the patients were nulliparous (72.4\%). Of the 2 patients who did not present with infertility, 1 presented with dysfunctional uterine bleeding. She was a 38 years old para $4^{+1}$ lady who had normal findings at hysteroscopy. The other was a 44 years old para $5^{+3}$ lady who presented with recurrent spontaneous miscarriages. Hysteroscopy showed minimal uterine adhesions which would not account for the miscarriages. Cervical os appeared intact and a chromosomal cause was suspected. Being advanced in age and a grandmultipara she was equally 
counseled against further pregnancies but refused as it was customary where she comes from to deliver 12 times!

Adhesiolysis for intrauterine adhesions constituted 67.2\% of all the surgical procedures performed. This was much higher than the $16.6 \%$ reported at the Aga Khan hospital in Kenya. ${ }^{6}$ Majority of our patients (71.1\%) had previously terminated one or more pregnancies and these are usually unsafe abortions, as abortion is still illegal in Nigeria. The result could be an increase in uterotubal infections and hence adhesions. There is equally a high-rate of pelvic inflammatory disease in Nigeria. ${ }^{7}$

Prior to the advent of hysteroscopy, the diagnosis of intrauterine adhesions depended upon historic criteria, physical findings and laboratory data while the treatment consisted of an attempt to bluntly disrupt the adhesions by using a uterine sound or small curette. ${ }^{8}$ Presently the use of hysteroscopy for the diagnosis and treatment of intrauterine adhesions has been shown to be mandatory and is equally the preferred method for the treatment of intrauterine septa, removal of foreign bodies, polyps and submucous myomas. ${ }^{8,9}$ Unfortunately there is still a paucity of both instruments and hysteroscopic skills in Nigeria. As at the time of this study only six centers had a functional hysteroscope, serving a population of over 140 million people! Only one of the six centers is a public institution.

Of the 43 patients who had hysteroscopic adhesiolysis, majority (21 or 48.8\%) had IUCD inserted following adhesiolysis. An inert IUCD with a large surface area is generally recommended. ${ }^{10}$ Lippes loop will fit effectively into this description but is no longer being marketed. We usually improvise with a copper $\mathrm{T} 380 \mathrm{~A}$, with the copper element removed before insertion into the uterine cavity to prevent any inflammatory reaction that may be caused by the copper element. Foley's catheter has however been shown to be significantly superior to IUCD in terms of improvement in normal menses as it reduces the need for a repeat adhesiolysis. ${ }^{10}$ Fortunately, only 2 of the 21 patients needed repeat adhesiolysis. All the patients were placed on prophylactic antibiotics for 7 days while those with an indwelling intrauterine device had oral estrogen to promote a regrowth of the endometrium and later a progestogen for withdrawal bleeding. Although 23 patients had endometrial polyps at hysteroscopy, 21 had hysteroscopy guided polypectomy with the use of hysteroscopic forceps and scissors. In the other two patients, one had a blind curettage as the polyps were too numerous to be removed individually under hysteroscopic guidance. The other had a large solitary polyp which was removed blindly with an oven forceps. One submucous fibroid was successfully removed with a hysteroscopic scissors, it measured approximately $1.2 \times 1 \mathrm{~cm}$ and had no myometrial extension. A resectoscope is the preferred instrument of choice for submucous myomectomy but unfortunately was unavailable at the center during the study period. Fetal bones within the endometrial cavity were an interesting finding. Secondary infertility due to intrauterine fetal bones has been reported by various authors. ${ }^{11,12}$ All three patients had previous second trimester termination of pregnancies by dilatation and curettage between 3 and 25 years prior to presentation and were experiencing regular normal menstrual periods. One achieved a spontaneous pregnancy two months after the fetal bone retrieval and had a vaginal delivery of a $2.8 \mathrm{~kg}$ female baby. It was suspected at transvaginal ultrasound scan that one of the patients probably had some retained bones within the myometrial tissues following repeated attempts at uterine evacuation for suspected adhesions on hysterosalpingogram. Our suspicion was based on the finding of the same hyper echoic shadow within the endometrial cavity and myometrium at transvaginal ultrasound scan. Her last pregnancy was terminated at 16 weeks gestation, 25 years before presentation!

The complication rate was $1.2 \%$. This compares favorably with the $0.7 \%$ reported in Kenya ${ }^{6}$ and $0.28 \%$ by Jansen et al. ${ }^{3}$ The patient in our study was a 33 years old para $1^{+1}$ lady who had general anesthesia for combined hysteroscopy and laparoscopy. She developed apnea after the procedure for which she needed mouth to mouth resuscitation, cardiac massage and oxygen by face mask. Intermittent positive pressure ventilation was instituted by the anesthetist. She recovered fully an hour later.

Hysteroscopic diagnosis and treatment has become very important in patients with infertility. It is one of the safest and most easily acquired surgical skills in gynecology. ${ }^{1}$ Acquisition of the equipment and skills especially in the public hospitals in Nigeria are urgently needed to obviate the need for blind procedures like endometrial curettage within the endometrial cavity.

\section{REFERENCES}

1. Bradley LD. Cutting the risk of hysteroscopic complications. Obg management 2004;16(1):1-12.

2. Bradley LD, Widrich T. Flexible hysteroscopy a state-of-theart procedure for gynaecologic evaluation. J Am Assoc Gynaecol Laparosc 1995;2:263-67.

3. Jansen FW, Vredevoogd CB, Ulzen K, Hermans J, Trimbos JB, et al. Complications of hysteroscopy: A prospective, multicentre study. Obstet Gynaecol 2000;96:517-20.

4. Konig M, Meyer A, Aydeniz B, Kurek R, Wallwiener. Hysteroscopic surgery: Complications and their prevention. Contrb Gynaecol Obstet 2000;20:161-70.

5. Valle RF. Hysteroscopy in the evaluation of female infertility. Am J Obstet Gynaecol 1980 June 15;137(4):425-31.

6. Parkar RB, Thagana NG. Hysteroscopic surgery at the Aga Khan Hospital, Nairobi. East Afr Med J 2004;81(7):336-40.

7. Bello TO. Pattern of tubal pathology in infertile women on hysterosalpingography in Illorin Nigeria. Annals of African Medicine 2004;3(2):77-79.

8. March CM. Hysteroscopy as an aid to diagnosis in female infertility. Clin Obstet Gynaecol 1983;26(2):302-12. 
9. Duggal BS, Sharma RK, Tarneja P, Rath SK, Wadhwa RD. Fertility enhancing hysteroscopic surgery: Our experience. MJAFI 2003;59:29-31.

10. Orhue AAE, Aziken ME, Eigbefoh JO. A comparison of two adjunctive treatments for intrauterine adhesions following lysis. Int J Gynecol Obstet 2003;82:49-56.
11. Melvis FA, Thomas MJ, Theodose CN. Prolonged retention of intrauterine bones. Obstet Gynecol 1991;78:919-20.

12. Dawood YM, Tasret JC. Prolonged retention of fetal bone after abortion causing infertility. Am J Obstet and Gynecol 1982;143:715-18. 